\title{
The Determination of Sucrose Content in Maple Sap Using Time- Domain Magnetic Resonance
}

\author{
Michael Gill ${ }^{1} \cdot$ Benedict Newling ${ }^{1}$ (D)
}

Received: 13 July 2021 / Accepted: 26 January 2022 / Published online: 14 February 2022

(C) The Author(s), under exclusive licence to Society for Sugar Research \& Promotion 2022

\begin{abstract}
The natural variation of sucrose concentration in maple tree sap is investigated using time-domain magnetic resonance (MR). The current study, which includes a concise introduction to the relevant MR properties, is a demonstration of principle showing how the relaxation time constant $T_{2}$ and the self-diffusion coefficient relate to the amount of sucrose and ionic content present in the collected sap samples. $T_{2}$ and self-diffusion coefficient for maple saps from six different trees, each sampled weekly in the spring of 2019, were measured using MR. The results were plotted against the sucrose concentration of each sample with the aim of determining if either quantity could serve as the basis for a non-invasive sucrose measurement for maple trees. The $T_{2}$ relaxation time constant was found not to be a reliable proxy for sucrose content in maple sap as it showed sensitivity to the slight changes in sap chemistry throughout the season and natural variation from tree to tree. The diffusion coefficient, determined through a standard pulsed-gradient spin-echo experiment, was insensitive to the changes in sap chemistry and showed a strong relationship to sucrose content. A diffusion measurement is thus proposed as the most suitable candidate for a non-invasive sucrose measurement for maple tree sap.
\end{abstract}

Keywords Maple - Sap · MR - Relaxation - Diffusion · Sucrose

Benedict Newling

bnewling@unb.ca

1 Department of Physics, University of New Brunswick, Fredericton, New Brunswick, Canada

\section{Introduction}

The production of maple syrup and related confections is an important industry in Eastern Canada with total production in 2019 having a value of over $\$ 500$ million CAD (Government of Canada 2020). The processing of maple sap into maple syrup, in which water is removed from sap through reverse osmosis and boiling to bring the overall sugar content from $\sim 2 \%$ (pure sap) to $\sim 64 \%$ (commercial syrup), is time and energy intensive. Within this process, any increase in efficiency can have a very significant impact on profitability. The combination of high production costs and a premium finished product means that the maple industry is prone to intentional mislabelling and counterfeiting. Differentiating between genuine and fake syrup is an important component of the enforcement of industry standards (Clément et al. 2014).

Maple sap is a solution that ranges in sugar concentration from 1 to $5 \%$ and water content from 95 to $99 \%$. Variations of sugar content depend on the tree, environmental conditions during growth, and time of year, with sucrose making up between 96 and $99 \%$ of the total sugars in pure sap (Perkins and van den Berg 2009). Complete analysis of sap chemistry identifies seasonal variation of sugars and other dissolved solids such as minerals and phenolic compounds, all in trace amounts (Lagacé et al. 2015).

While the total sugar content in tree sap varies due to external conditions, the relative sweetness of one tree with respect to its neighbouring trees has been shown to be consistent; a sweet tree is always a sweet tree (Taylor 1956). An obvious potential avenue to improve the efficiency of the syrup process is to collect sap only from the sweetest trees, and if possible, identify and encourage the sweetest maple trees in the growth stage of a maple stand. 
The standard approach to testing the sweetness of sap relies on refractometry. The sapwood is punctured, allowing for the extraction of a sample to be tested in a refractometer. Because this process requires some damage to the tree, there is a lower limit to the age and size of trees that can withstand the stress of the procedure. For the collection of large amounts of sap in the case of syrup production, only trees of at least $25 \mathrm{~cm}$ diameter at breast height are tapped (Perkins and van den Berg 2009). In order to collect small volumes of sap from smaller trees for testing, a process called micro-tapping was developed. However, this newer procedure is still invasive and is only rarely implemented in trees 3 years of age with a stem diameter of $1-2 \mathrm{~cm}$. Micro-tap testing is usually done once trees are 5-7 years old (Staats 1992).

The benefit of early sugar testing of saplings extends beyond the commercial sugarbush. There have been a number of large progeny studies, with an experimental timescale of 40 years, the goal of which was to research the possibility of producing a sweeter nurse stock of maple trees through select breeding. Due to pests, weather, and human activity, many of these trees did not survive long enough to be tested, limiting the reliability of the eventual study results (Staats 1992). A completely non-invasive sugar test that could be implemented in the youngest of saplings would be a powerful new tool in these and other types of studies.

The continued development of low-cost and portable magnetic resonance (MR) sensors opens a new horizon of measurements that can be used to study and understand industrial and agricultural production (Capitani et al. 2017; Blümich et al. 2009). As yet, the authors are unaware of any peer-reviewed studies of the MR properties of maple sap; however, there have been numerous studies

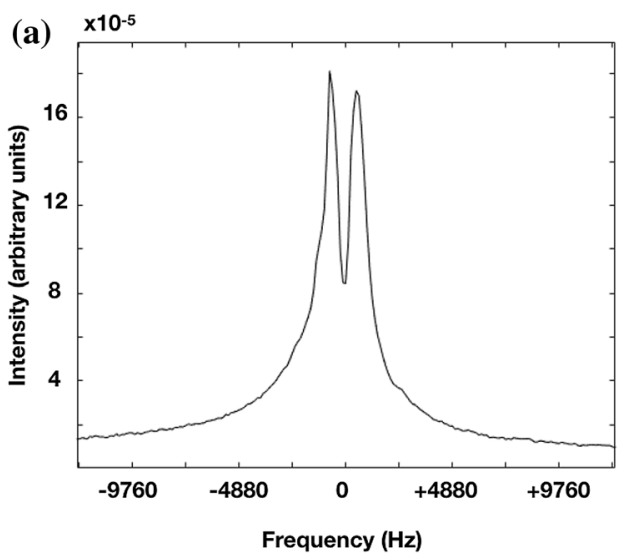

Fig. 1 Fourier spectra taken on a $100 \mathrm{MHz}$ superconducting MR scanner. The vertical axes are not comparable. The two spectra represent solutions of different concentrations and different sized vials. a Sucrose- $\mathrm{D}_{2} \mathrm{O}$ solution: the two, low-resolution peaks on other sucrose solutions, and the aim of this paper is to differentiate the MR behaviour of natural maple sap from that of a pure sucrose solution and to describe a reliable method for assessing sucrose content of sap using MR.

\section{MR Background}

Unlike MR spectroscopy, which has a line width resolution to distinguish each hydrogen environment in a given molecule, the resolution of typical time-domain MR equipment is insufficient to characterize a molecule based on its chemical shift, as demonstrated in Fig. 1. In lieu of directly observing the spectrum, time-domain MR monitors the decay of signal following one or more radio frequency (RF) pulses.

There are two fundamental lifetimes in MR: the spinlattice relaxation time $\left(T_{1}\right)$, which describes a sample's return to thermal equilibrium; and the spin-spin relaxation time $\left(T_{2}\right)$, which describes the loss of signal due to dephasing of neighbouring "spins" $\left({ }^{1} \mathrm{H}\right.$ nuclei in the present measurements). Since loss of signal can also stem from the Brownian motion of molecules within the sample itself, time-domain MR also provides a means to measure molecular self-diffusion, typically reported as a diffusion coefficient $(D)$.

The time interval between successive measurements, commonly referred to as either the last delay (LD) or the repetition time (TR), needs to be sufficiently long to allow the sample to return to thermal equilibrium to ensure no residual magnetization is carried over, ensuring that each measurement yields an independent result. The standard $\mathrm{LD}$ is equal to $5 \times T_{1}$ of the sample.

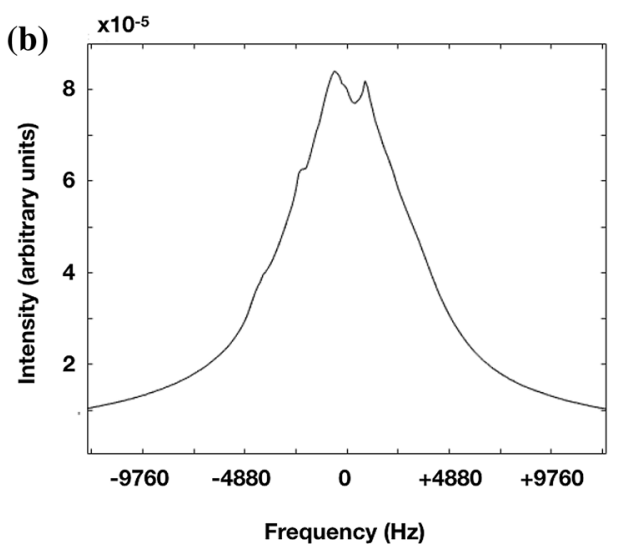

correspond to the backbone hydrogen on the sucrose molecule and the $\mathrm{OH}$ groups. b Sucrose $-\mathrm{H}_{2} \mathrm{O}$ solution: the spectral peaks relating directly to the sucrose molecule, as seen in $1 \mathbf{a}$, are dwarfed by the bulk water peak 
When paramagnetic ions, such as $\mathrm{Cu}^{2+}$ and $\mathrm{Mn}^{2+}$, are in solution, the presence of multiple varying local magnetic fields causes a shortening of both characteristic times $T_{1}$ and $T_{2}$ of a solution due to more rapid dephasing of magnetic spins ( $T_{2}$ effect) and an increase in the relaxation efficiency of nearby water protons due to long-range electron relaxation of the paramagnetic ions $\left(T_{1}\right.$ effect) (De León-Rodríguez et al. 2015). These effects increase linearly with respect to the inverse of $T_{1}$ and $T_{2}$ with increasing concentration of paramagnetic ions (Parigi and Luchinat 2018). Since diffusion is the process of random molecular motion, it is unaffected by the presence of paramagnetic ions.

At relatively low concentrations of sucrose, the MR signal is dominated by water, as can be seen in the Fourier spectrum in Fig. 1b, and as such the sample will have a single $T_{2}$ value (compared to mixtures such as oil and water, which have two or more measured $T_{2}$ values). But by virtue of the sucrose being dissolved, the water neighbouring the sucrose molecule will form a hydration shell or layer, often referred to as "bound water", the population of which will be directly proportional to the concentration of sucrose. Thus, the measured $T_{2}$ will be a weighted average of that of the free water and the bound water and is described by the following equation:

$\frac{1}{T_{2 \text { obs }}}=\frac{a}{T_{2 \text { free }}}+\frac{b}{T_{2 \text { bound }}}$,

where $a$ and $b$ are the concentrations of free water and bound water, respectively. Since $a$ and $b$ must sum to 1 , we can rewrite the equation as

$\frac{1}{T_{2 \text { obs }}}=\frac{1}{T_{2 \text { free }}}-\frac{b}{T_{2 \text { free }}}+\frac{b}{T_{2 \text { bound }}}$.

Finally, noting that the concentration of bound water is much less than 1, we can approximate the second term by zero, which yields an expression connecting the inverse of our observed $T_{2}$ and the concentration of sucrose

$\frac{1}{T_{2 \mathrm{obs}}}=\frac{1}{T_{2 \text { bound }}} \cdot b+\frac{1}{T_{2 \text { free }}}$.

Previous MR experiments involving sucrose solutions have all reported this linear relationship (Aroulmoji et al. 2012; Fabri et al. 2005; Ziegler et al. 2003; Eustace and Jordan 1995).

\section{Material and Methods}

Sap was collected from six maple trees in rural New Brunswick (Canada) for four consecutive weeks during March 2019 and kept frozen after initial measurements. The 11th March samples were spoiled due to a freezer malfunction before the diffusion coefficients could be measured; however, those samples were viable during the relaxation phase of the experiment. At the time of spoilage, only the 11th March samples were in the freezer; the remaining samples were kept in a chemical fridge and were unaffected as a result.

Samples were collected at each tree by manually transferring sap from buckets, which had been emptied the previous day after sap flow had ceased, into unused plastic sample vials. This was to ensure sap tested was entirely the product of a single day's run. Sucrose was measured in the field at point of collection and again in laboratory before storage. All six trees were sampled at each of the four time points, and a second stem of one tree was sampled three times beginning in week 2 for a total of 27 individual samples.

The mineral content of the non-spoiled sap samples was determined by ICP/ES with the assistance of the UNB Civil Engineering wastewater testing laboratory using a Varian Vista MPX Axial ICP ES (Inductively Coupled Plasma Emission Spectrometer) instrument. Detected wavelengths (nm) were as follows: $\mathrm{Ca}$ (396.847); $\mathrm{Cu}$ (327.395); Fe (259.940); K (769.897); Mg (280.270); Mn (294.921); Na (589.592); P (214.914); and $\mathrm{Zn}$ (206.200). Testing for $\mathrm{Ca}$, $\mathrm{K}$ and $\mathrm{Mg}$ required a 20 -fold dilution with distilled water, whereas the remaining elements were determined with unadulterated sap.

Sucrose content is given in Brix per cent $(\% \mathrm{Bx})$ as measured by a Hanna Instruments portable, digital refractometer, HI 96,801 (Woonsocket, RI), with a resolution of $0.1 \% \mathrm{Bx}$ and precision of $\pm 0.2 \% \mathrm{Bx}$.

The diffusion and relaxation experiments were conducted on a Nalorac (Martinez, CA) $2.4 \mathrm{~T} 32 \mathrm{~cm}$ i.d. horizontal bore superconducting magnet with a watercooled 7.5-cm Nalorac gradient set driven by Techron (Elkhart, IN) 8710 amplifiers. The RF probe was a homemade $4.5-\mathrm{cm}$ i.d. birdcage probe driven by a $2 \mathrm{~kW}$ Tomco (Tomco Technologies, Stepney, Australia) RF amplifier. The console was a Tecmag (Houston, TX) Redstone.

Spin-spin $\left(T_{2}\right)$ lifetimes were measured using the CarrPurcell-Meiboom-Gill (CMPG) sequence with an echo time of $1 \mathrm{~ms}$ and $90^{\circ}$ and $180^{\circ} \mathrm{RF}$ pulse durations of $15 \mu \mathrm{s}$ and $30 \mu \mathrm{s}$, respectively. While not included in our results, the spin-lattice $\left(T_{1}\right)$ relaxation rate was measured using a standard inversion recovery (IR) pulse sequence using the same RF pulse lengths already noted (Callaghan 2011). Each $T_{2}$ measurement took approximately $2 \mathrm{~min}$.

The gradient strength in the diffusion measurements was calibrated against distilled water at $14{ }^{\circ} \mathrm{C}$ using $1.7156 \times 10^{-9} \mathrm{~m}^{2} / \mathrm{s}$ for the value of the diffusion coefficient, as calculated by the equation given in Holz et al. (2000). These measurements were done using a standard pulsed-gradient spin-echo (PGSE) sequence (Callaghan 
2011). The maximum gradient strength was $0.278 \mathrm{~T} / \mathrm{m}$ with $\Delta=30 \mathrm{~ms}$ and $\delta=4 \mathrm{~ms}$, and the overall measurement time was approximately $7 \mathrm{~min}$.

Temperature control was achieved by resting samples in a cool air stream generated from nitrogen boil-off controlled with an Omega (Omega Engineering, Stamford, CT) 6002 temperature controller that was stable to $\pm 0.5^{\circ} \mathrm{C}$. Sample temperature was measured before and after measurement using a standard immersible alcohol thermometer with a range of $-20^{\circ} \mathrm{C}$ to $110^{\circ} \mathrm{C}$ with a precision of $\pm 0.5^{\circ} \mathrm{C}$.

Horizontal error bars shown in Fig. 3 reflect the precision of the refractometer, while the vertical error bars show the standard deviation of multiple measurements of the diffusion coefficient.

In addition, low-field relaxation experiments were conducted on a $10 \mathrm{MHz}$ Bruker Minispec MQ10 NMR Analyzer (Rheinstetten, Germany) using the CPMG sequence with an echo time of $10 \mathrm{~ms}$ and $90^{\circ}$ and $180^{\circ} \mathrm{RF}$ pulse duration of $2.08 \mu \mathrm{s}$ and $4.38 \mu \mathrm{s}$.

\section{Results}

Two types of artificial saps were made by diluting pure sucrose, produced by Sigma-Aldrich, and commercially available maple sugar, produced by Acadia Maple in Nova Scotia, in distilled water to achieve a representative concentration of $1-5 \%$ sucrose. The sucrose concentration of an actual sap sample was also varied with distilled water as an additional point of comparison. Figure 2 illustrates the relationship between relaxation lifetime and sucrose concentration. If the sucrose in the solutions were primarily responsible for affecting spin-spin relaxation, then each solution would have similar $T_{2}$ values. As can be seen in Fig. 2, however, this was not the case. The maple solutions relaxed faster than pure sucrose. Other solutions (for example a second source of maple sugar) gave additional results consistent with Fig. 2.

Because chemical composition of sap is known to vary both from tree to tree and over time, saps from six maple trees were collected over 4 weeks in March 2019. The $T_{2}$ relaxation of these saps was measured and plotted against their measured sugar content. Again, if the variation of sucrose between samples was the principal cause of relaxation differences, the plot of relaxation versus sucrose content would produce a linear plot, regardless of other natural variations in sap chemistry. The scatter plot shown in Fig. 3a and $\mathrm{b}$ demonstrates relaxation behaviour that is attributable to something other than just sucrose and that this variability is not affected by the field strength of the MR scanner. The $T_{1}$ relaxation of these samples was also measured and showed similar scatter as that in Fig. 3.

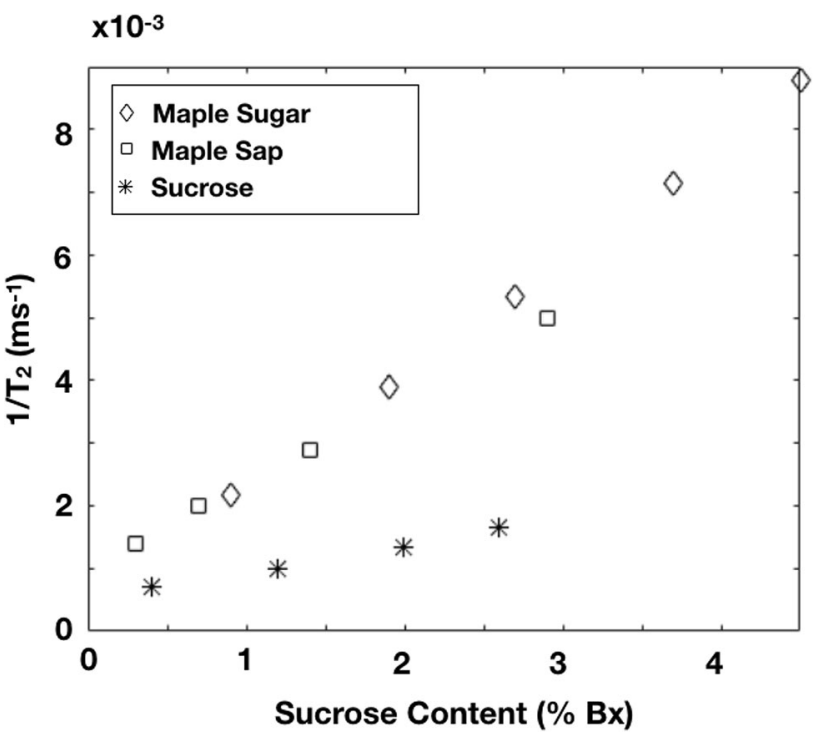

Fig. $2 T_{2}$ relaxation plotted against sucrose content (measured with a refractometer) for three types of sugar solutions. Note that the sap is diluted with distilled water, which gives rise to a $1 / T_{2}$ that varies linearly with increasing sucrose content, consistent with pure sucrose solutions

Table 1 shows the mineral analysis results, which were consistent with previously published reports of maple sap content (Perkins and van den Berg 2009; Lagacé et al. 2015). $\mathrm{Cu}$ and $\mathrm{Fe}$ were detected weakly at concentrations near to and below the detection limit of the instrument. $\mathrm{Cu}$ is included in Table 1 because it was at detectable concentration levels in all but five samples, whereas $\mathrm{Fe}$ is excluded because it appeared at the detection limit in only five of the twenty samples. Owing to its strong magnetic properties, it is important to mention within the context of a MR study.

Since $\mathrm{Mn}^{2+}$ is an effective relaxation agent and is found in significant, highly variable concentrations in maple sap, both in the literature and in the current study, the measured spin-spin relaxation of the sap samples is plotted against their manganese concentration (Fig. 4). The plot shows a convincingly linear relationship between manganese concentration and the inverse of $\mathrm{T}_{2}$ with an $\mathrm{R}$-squared value of 0.7667 .

Next, the water diffusion coefficients of the viable sap samples were measured. The 18 March and 25 March saps were measured on separate days during the summer of 2019 during which the ambient temperature of the laboratory varied substantially. Even with careful temperature control, we were unable to conduct both sets of measurements at the same temperature; however, each measurement within both sets was the same within the precision of the thermometer. Temperature control was necessary because molecular self-diffusion is highly sensitive to temperature (Holz et al. 2000). In fact, the measured 


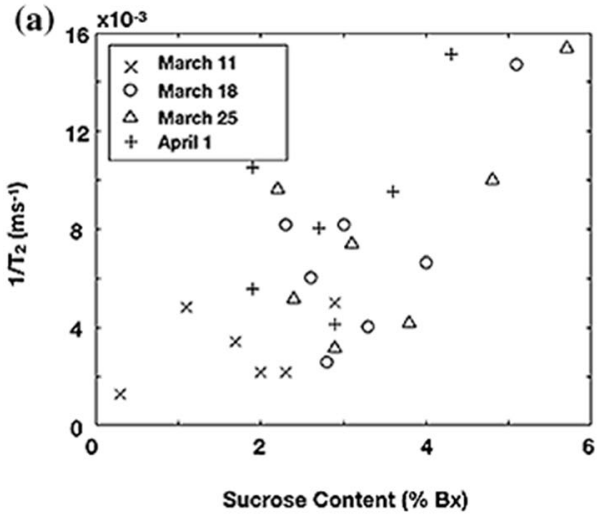

Fig. $3 T_{2}$ relaxation plotted against sucrose content for maple sap taken from six different trees sampled over a 4-week period in the spring of 2019. Beginning in the second trial week, a second tap was added to a multistem tree yielding an additional data point.

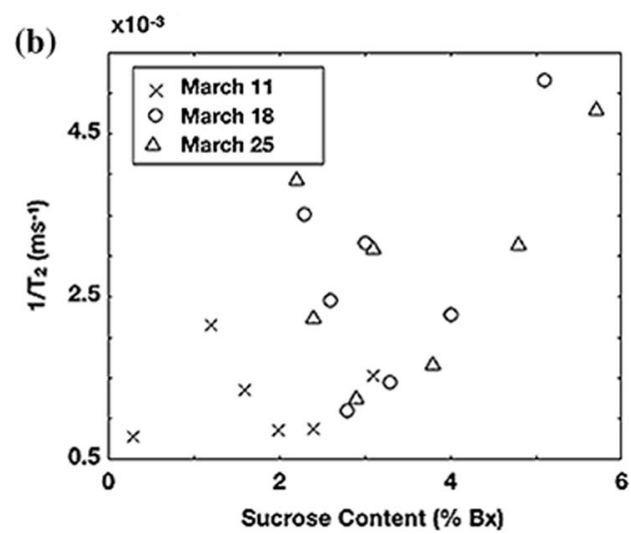

Measurements taken at a $100 \mathrm{MHz}$ and b $10 \mathrm{MHz}$. A mechanical breakdown in the $10 \mathrm{MHz}$ instrument delayed the measurement of the final set of sap samples, and the instrument was decommissioned before measurements could be completed

Table 1 Summary of mineral content in tested sap

\begin{tabular}{lllcc}
\hline Element & Average $(\mathrm{mg} / \mathrm{L})$ & Minimum $(\mathrm{mg} / \mathrm{L})$ & Maximum $(\mathrm{mg} / \mathrm{L})$ & Standard deviation $(\mathrm{mg} / \mathrm{L})$ \\
\hline $\mathrm{Ca}$ & 16.49 & 2.81 & 44.39 & 12.30 \\
$\mathrm{Cu}$ & 0.020 & nd & 0.032 & 0.007 \\
$\mathrm{~K}$ & 85.80 & 25.16 & 204.53 & 48.10 \\
$\mathrm{Mn}$ & 2.81 & 0.32 & 9.05 & 2.21 \\
$\mathrm{Mg}$ & 3.00 & 0.49 & 11.29 & 2.61 \\
$\mathrm{Na}$ & 1.69 & 0.46 & 4.65 & 1.29 \\
$\mathrm{P}$ & 5.70 & 1.53 & 14.23 & 3.85 \\
$\mathrm{Zn}$ & 0.202 & 0.045 & 0.871 & 0.199 \\
\hline
\end{tabular}

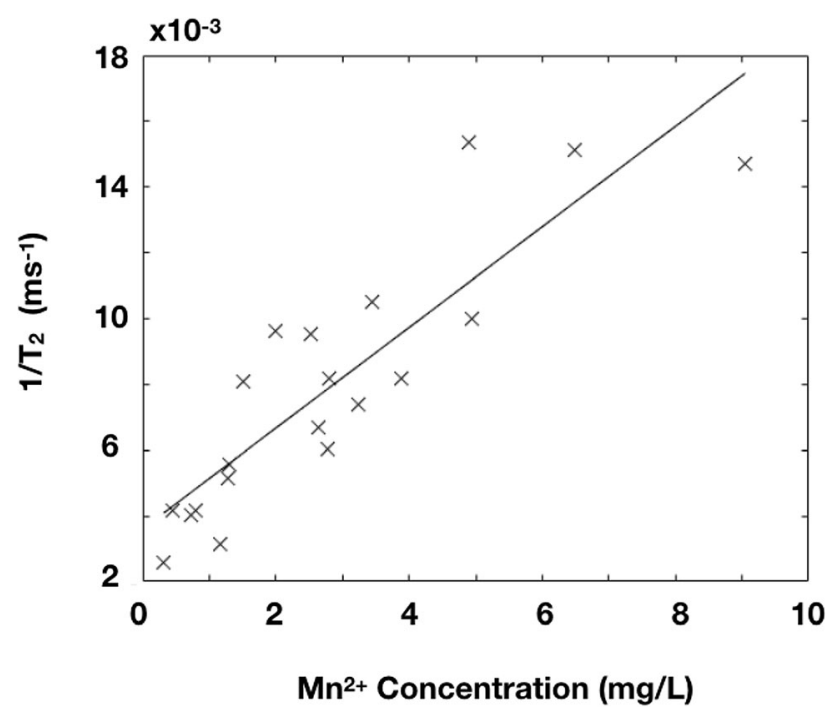

Fig. $4 T_{2}$ relaxation plotted against manganese content for maple sap for all sap samples that remained unspoiled at the time of ICP/ES analysis. Line of best fit has an $R$-squared value of 0.7667 change in diffusion coefficient from a $1 \%$ increase in sucrose concentration was less than the change brought about by a $1{ }^{\circ} \mathrm{C}$ increase in temperature as shown in Fig. 5 . Despite this issue in the relative sensitivity to temperature and sucrose concentration, Fig. 5 shows that self-diffusion of maple saps is dependent upon the sucrose concentration and not upon other changes in sap chemistry.

A previous study of the diffusion coefficient of water in sucrose solution identified an apparent exponential relationship (Aroulmoji et al. 2012); however, that study investigated a much larger range of sucrose concentrations ( $\sim 5.1 \%$ to $\sim 44.5 \%$, as read from their published figure). Since any exponential function is approximately linear at points near zero, we have chosen a straight-line fit for the current data. The 25th March set shows a stronger linear relationship than that of the 18th March, as evidenced by their $R$-squared values of 0.9194 and 0.7219 , respectively. It is not entirely surprising that the set with the smallest range of concentrations would yield a fit with the most uncertainty. In addition to the statistical quantity of $R$ squared, the reasonableness of a linear fit is supported by 


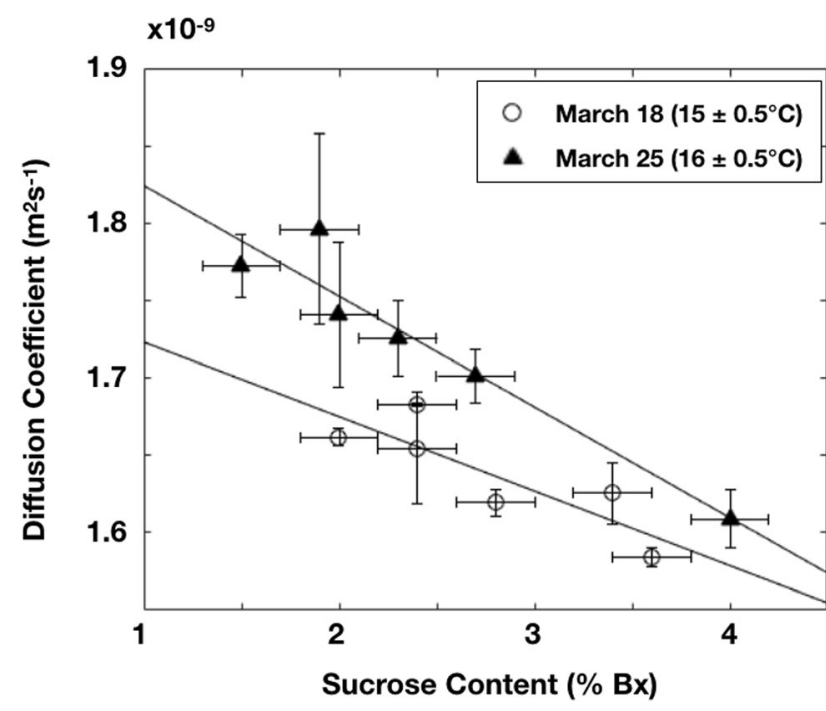

Fig. 5 Plot of the molecular self-diffusion coefficient of water in maple saps versus measured sucrose content. The volume of sap collected from the multistem tap was insufficient for all measurements and did not survive into the diffusion trials. The COVID-19 pandemic delayed testing of the fourth set of saps; then, the $100 \mathrm{MHz}$ magnet suffered a fatal quench before testing could be concluded. Sap samples used for the diffusion measurements were poured off from the partially thawed main samples used in previous experiments resulting in slightly different sucrose contents being reported between Figs. 3 and 5. $R$-squared values of the fit lines on the 18 March and 25 March data points were 0.7219 and 0.9194 , respectively

the extrapolated diffusion value of the $0 \%$ concentration point (the $y$-intercept), which should be the diffusion coefficient of water at the given temperature. In this case, both fit lines produce water diffusion values that are within the uncertainty range of values calculated based on $\mathrm{Holz}$ et al. (2000): $1.772 \times 10^{-9} \mathrm{~m}^{2} / \mathrm{s} \quad$ (fit) to $1.765 \pm 0.181 \times 10^{-9} \mathrm{~m}^{2} / \mathrm{s}$ (calculated) for 18 March, and $1.897 \times 10^{-9} \mathrm{~m}^{2} / \mathrm{s}$ (fit) to $1.8152 \pm 0.184 \times 10^{-9} \mathrm{~m}^{2} / \mathrm{s}$ (calculated) for 25 March.

\section{Discussion}

When taken alongside the apparent linear relationship between $\mathrm{Mn}^{2+}$ and measured relaxation (Fig. 4), the nonlinearity in Fig. 3a and b clearly demonstrates that any relaxation measurement of maple sap will only communicate information about paramagnetic concentrations present within the sap. The $R$-squared value of 0.7667 in Fig. 4 indicates that while manganese dominates the relaxation behaviour in maple sap, $T_{2}$ is still being affected by other paramagnetic ions and molecules. Even though the scope of the current study is limited in its sample size, large sap chemistry studies confirm that the variation seen in this study is typical (Lagacé et al.
2015). Therefore, one can reasonably expect that any attempt to correlate sucrose concentration with basic relaxation in maple sap is impossible without first having a detailed knowledge of the full chemistry of the particular sap sample. This observation could be exploited to form a useful measurement for the detection of fake/counterfeit maple syrup.

Since the relaxation times of maple saps were highly sensitive to the natural variation of paramagnetic ions, the measurement of $T_{2}$ could provide a quick and simple way to differentiate pure maple syrup from artificial syrups. As sap is transformed into syrup, a substance called "niter" or "sugar sand" forms and falls out of solution. The composition of this precipitant is highly variable and does contain small amounts of copper and manganese (Perkins and van den Berg 2009). The fact that some manganese survives this process can be inferred from Fig. 2, which shows that diluted maple sugar (a maple product made by continued heating of pure syrup) has a comparable relaxation curve as maple sap, both being distinct from a simple sucrose solution. A standard Brix measurement and a value for the $T_{2}$ of the syrup in question would be enough to locate it on either a sucrose calibration curve for artificial syrups or within a typical range of genuine maple syrup.

The water self-diffusion coefficient is not affected by the presence of paramagnetic ions, and so the seemingly linear lines of best fit in Fig. 5 are not unexpected. Since the concentration of all dissolved solids in sap pales in comparison with sucrose, the molecular motion of $\mathrm{H}_{2} \mathrm{O}$ is most affected by the increased viscosity caused by the dissolved sucrose. Because the mineral composition and sucrose content of the saps tested in the current study were representative of general maple sap, the observed trend in Fig. 5 ought to be generalizable as well. In order to more fully define the functional relationship between diffusion coefficient and sucrose concentration in maple sap, other more extensive studies will need to be conducted with a broader range of sucrose contents and across a larger variation of experimental temperatures-an endeavour far beyond the scope and limited sample size of the current study.

Any attempt at exploring and mapping the relationship between sucrose and diffusion in maple sap will need to deal with the extreme temperature dependence of the diffusion process. The temperature control and monitoring in the current study was limited to $0.5{ }^{\circ} \mathrm{C}$. Owing to the wide range of sucrose content present in the saps tested, particularly the 18th March set, the correlation between sucrose and diffusion was not overshadowed by the coarse temperature control.

The time required to measure a sample's diffusion coefficient is largely constrained by two factors: the number of gradient values used and the LD of the sequence. In 
our measurements, 11 different gradient values were used to provide the highest confidence in our reported values. In practice, however, fitting of the data was excellent and the measurement could have been performed with fewer gradient steps. By following the method of Stait-Gardner et al. (2008), the diffusion measurement can occur at the steadystate magnetization instead of waiting for the return to thermal equilibrium after each iteration. The added measurement speed becomes a trade-off with the overall accuracy of the measurement; however, with care, a suitable balance can be found. By implementing the aforementioned changes, this 7-min measurement could be accomplished in as little as $2 \mathrm{~min}$.

A planned follow-up study will see sets of cuttings of young coppice wood (diameter less than $5 \mathrm{~mm}$ ) tested using a portable MR device in order to confirm that changes in self-diffusion can be observed in plant cuttings. The study will employ a permanent magnet sensor with a constant gradient (Ross et al. 2021) which will simplify the measurement of diffusion by eliminating the need to have well-controlled and defined magnetic field gradients.

\section{Conclusion}

Two magnetic resonance measurements were performed on extruded vials of maple sap to determine how the MR signal relates to the sucrose concentration of maple sap. The spin-spin relaxation time constant, $T_{2}$, was heavily influenced by the natural variations in paramagnetic ions in the sap, and those effects dominated over the effect of sucrose concentration. The results shown in Fig. 1 imply that the presence of paramagnetic ions, particularly manganese, in sap could be exploited, allowing the differentiation between real and artificial maple syrup using $T_{2}$. The diffusion coefficient of maple sap was found to decrease as sucrose concentration increased, meaning that diffusion could be used as a proxy for sucrose given a well-defined calibration curve built upon further clinical trials. Since MR is, by nature, non-invasive, these findings can be developed into non-invasive/non-destructive measurements of sugar content, potentially benefiting the maple industry at both the forest planning and product fidelity levels.

Acknowledgements The authors are grateful for the insights of Drs. Bruce Balcom, Bryce MacMillan and Dennis Connor and for financial support from the New Brunswick Innovation Foundation (RAI 2019-038).

\section{References}

Aroulmoji, Vincent, Mohammed Mathlouthi, Luigi Feruglio, Erminio Murano, and Mario Grassi. 2012. Hydration properties and proton exchange in aqueous sugar solutions studied by time domain nuclear magnetic resonance. Food Chemistry 132: 1644-1655. https://doi.org/10.1016/j.foodchem.2011.01.110.

Blümich, Bernhard, Jörg. Mauler, Agnes Haber, Juan Perlo, Ernesto Danieli, and Federico Casanova. 2009. Mobile NMR for geophysical analysis and materials testing. Petroleum Science. 6: 1-7. https://doi.org/10.1007/s12182-009-0001-4.

Callaghan, Paul. 2011. Translational dynamics and magnetic resonance. Oxford: Oxford University Press.

Capitani, Donatella, Anatoly P. Sobolev, Valeria Di Tullio, Luisa Mannina, and Noemi Proietti. 2017. Portable NMR in food analysis. Chemical and Biological Technologies in Agriculture. https://doi.org/10.1186/s40538-017-0100-1.

Clément, Alain, Bernard Panneton, Nathalie Martin, Luc Lagacé. 2014. Maple syrup quality control. Canadian Food Insights Summer: 34-35.

Eustace, C.A., and R.B. Jordan. 1995. Sugar content evaluation using Earth's field proton magnetic resonance. American Society of Agricultural Engineers. 38 (5): 1563-1571.

Fabri, Deborah, Martin A.K.. Williams, and Thomas K. Halstead. 2005. Water $\mathrm{T}_{2}$ relaxation in sugar solutions. Carbohydrate Research 340: 889-905. https://doi.org/10.1016/j.carres. 2005.01.034.

Government of Canada. 2020. Statistical Overview of the Maple Industry 2019. https://multimedia.agr.gc.ca/pack/pdf/maple_ erable_2019a-eng.pdf. Accessed 15 April 2021.

Holz, Manfred, Stefan R. Heil, and Antonio Sacco. 2000. Temperature-dependent self-diffusion coefficients of water and six selected molecular liquids for calibration in accurate ${ }^{1} \mathrm{H}$ NMR PFG measurements. Physical Chemistry Chemical Physics 2: 4740-4742. https://doi.org/10.1039/B005319H.

Lagacé, Luc, Simon Leclerc, Carmen Charron, and Mustapha Sadiki. 2015. Biochemical composition of maple sap and relations among constituents. Journal of Food Composition and Analysis. 41: 129-136. https://doi.org/10.1016/j.jfca.2014.12.030.

De León-Rodríguez Luis, M., André F. Martins, Marco C. Pinho, Neil Rofsky, and A. Dean Sherry. 2015. Basic MR relaxation mechanisms and contrast agent design. Journal of Magnetic Resonance Imaging. 42: 545-565.

Parigi, Giacomo, and Claudio Luchinat. NMR Consequences of the Nucleus-Electron Spin Interactions. In Paramagnetism in experimental biomolecular NMR, eds. Claudio Luchinat, Giacomo Parigi, and Enrico Ravera, pp. 1-41. London: Royal Society of Chemistry.

Perkins, Timothy D., and Abby K. van den Berg. 2009. Maple syrupproduction, composition, chemistry, and sensory characteristics. Advances in Food and Nutrition Research. 56: 101-143.

Ross, Michael M.B., Grant R. Wilbur, Prisciliano F.J. Cano, de Barrita, and Bruce J. Balcom. 2021. A portable, submersible, MR sensor-The Proteus magnet. Journal of Magnetic Resonance. 326: 1-8. https://doi.org/10.1016/j.jmr.2021.106964.

Staats, Lewis J. 1992. Progeny testing 10-year old sugar maple families for sugar sap concentration. M.Sc. thesis. State University of New York.

Stait-Gardner, Tim, P.G. Anil Kumar, and Willian S. Price. 2008. Steady state effects in PGSE NMR diffusion experiments. 
Chemical Physics Letters 462: 331-336. https://doi.org/10.1016/ j.cplett.2008.07.084.

Taylor, Fred H. 1956. Variation in sugar content of maple sap. Vermont Agricultural Experiment Station Bulletin. 587: 3-39.

Ziegler, Gregory R., Bryce MacMillan, and Bruce J. Balcom. 2003. Moisture migration in starch molding operations as observed by magnetic resonance imaging. Food Research International 36: 331-340. https://doi.org/10.1016/S0963-9969(02)00224-7.

Publisher's Note Springer Nature remains neutral with regard to jurisdictional claims in published maps and institutional affiliations. 